\title{
Tokyo Axion Helioscope
}

\author{
Makoto Minowa \\ Department of Physics and RESCEU, the University of Tokyo, 7-3-1 Hongo, Bunkyo-ku, Tokyo \\ 113-0033, Japan
}

\begin{abstract}
The idea of a magnetic axion helioscope was first proposed by Pierre Sikivie in 1983. Tokyo axion helioscope was built exploiting its detection principle with a dedicated cryogen-free superconducting magnet and PIN photodiodes for x-ray detectors. Solar axions, if exist, would be converted into x-ray photons in the magnetic field. Conversion is coherently enhanced even for massive axions by filling the conversion region with helium gas. Its start up, search results so far and prospects are presented.
\end{abstract}

Keywords: solar axion, helioscope, superconducting magnet

PACS: $14.80 . \mathrm{Mz}, 07.85 . \mathrm{Fv}, 96.60 . \mathrm{JW}$

\section{SOLAR AXION DETECTION, THE PRINCIPLE}

Existence of the axion is implied to solve the strong CP problem of Quantum chromodynamics (QCD)[1, 2, 3, 4]. The axion would be produced in the solar core through the Primakoff effect. It can be converted back to an x-ray in a strong magnetic field in the laboratory by the inverse process. This is the principle of the solar axion detection by the axion helioscope and appeared for the first time in the paper[5] entitled "Experimental Tests of the 'invisible' axion" written by Pierre Sikivie in 1983, 27 years ago. Sikivie indeed mentioned the idea of the axion helioscope in this paper.

The conversion process is coherent when the axion and photon remain in phase over the length of the magnetic field[8] if the axion is almost massless. Coherence can be maintained even for higher mass axions if the conversion region is filled with a low-Z buffer gas like helium[7, 8].

\section{EXPERIMENTAL EFFORTS}

A possible laboratory detector design was first proposed by K. van Bibber et al.[7], and there was a pioneering helioscope experiment by Lazarus et al.[6] in 1992. It was the first solar axion search experiment using magnetic field in a laboratory. At that time, however, a fixed magnet was used and running time was short.

They put significant upper limits on $g_{a \gamma \gamma}$ for the axion mass less than $0.11 \mathrm{eV}$. The measurements were done with vacuum magnetic field region for the lower axion mass region and with a buffer helium gas of up to 100 Torr for the higher mass region.

We started the construction of Tokyo axion helioscope in 1995, and the phase 1 result[9] was published in 1998 without a buffer gas in the manetic field region. Then, phase 2 measurement for the axion mass less than $0.27 \mathrm{eV}$ with a helium gas was made and result was published in 2002[10]. 
In the mean time, CAST experiment[12] was getting started in CERN and published their result for measurements with vacuum magnetic field region.

The third phase of Tokyo axion helioscope was then prepared, and the result was published for the axion mass region between $0.84 \mathrm{eV}$ and $1.00 \mathrm{eV}$ in 2008[11]. It is the first result to search for the axion in the $g_{a \gamma \gamma}-m_{a}$ parameter region of the preferred QCD axion models with a magnetic helioscope.

Almost at the same time, CAST published their new results[13] with a helium-4 buffer gas in the magnetic field region.

\section{TOKYO AXION HELIOSCOPE}

The schematic view of the Tokyo axion helioscope is shown in Fig. 1. Its main components are common to phase 1 through 3 of the Tokyo axion helioscope experiments[9, $10,11]$. It is designed to track the sun in order to achieve long exposure time. It consists of a dedicated superconducting magnet, X-ray detectors, a gas container, and an altazimuth mounting.

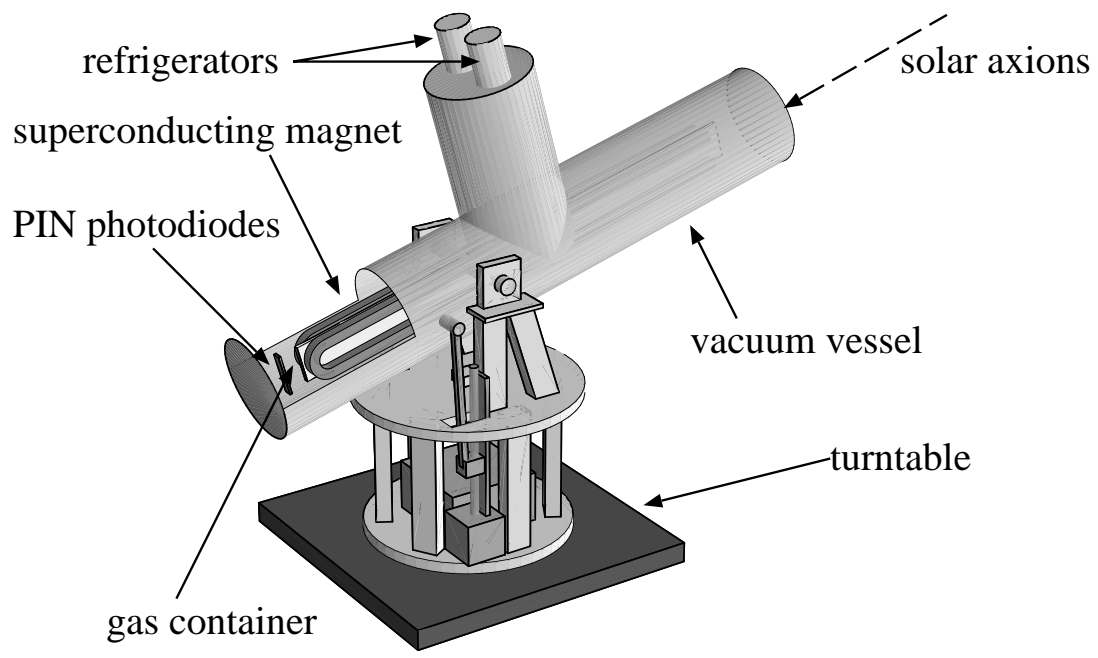

FIGURE 1. Tokyo axion helioscope

The superconducting magnet[14] consists of two 2.3-m long race-track shaped coils running parallel with a $20-\mathrm{mm}$ wide gap between them. The magnetic field in the gap is $4 \mathrm{~T}$ in the direction perpendicular to the helioscope axis. The coils are kept at $5-6 \mathrm{~K}$ during operation. The magnet was made cryogen-free by making two Gifford-McMahon refrigerators to cool it directly by conduction, and is equipped with a persistent current switch. Thanks to these features, the magnet can be freed from thick current leads after excitation, and the magnetic field is very stable for a long period of time without supplying current. The container to hold dispersion-matching buffer gas is inserted in the $20 \times 92 \mathrm{~mm}^{2}$ aperture of the magnet. Its body is made of four $2.3-\mathrm{m}$ long $0.8-\mathrm{mm}$ thick stainless-steel square pipes welded side by side to each other. Sixteen PIN photodiodes, Hamamatsu Photonics S3590-06-SPL, are used as the X-ray detectors [15], whose chip sizes are $11 \times 11 \times 0.5 \mathrm{~mm}^{3}$ each. The effective area of a photodiode was measured formerly using a pencil-beam X-ray source, and found to be larger than $9 \times 9 \mathrm{~mm}^{2}$. It 
has an inactive surface layer of $0.35 \mu \mathrm{m}$ [16]. The entire axion detector is constructed in a vacuum vessel and the vessel is mounted on an altazimuth mount. Its trackable altitude ranges from $-28^{\circ}$ to $+28^{\circ}$ and its azimuthal direction is designed to be limited only by a limiter which prevents the helioscope from endless rotation. However, in the present measurement, the azimuthal range is restricted to about $60^{\circ}$ because a cable handling system for its unmanned operation is not completed yet.

\section{RESULTS}

Fig. 2 shows the upper limit to $g_{a \gamma \gamma}$ plotted as a function of $m_{a}$. Our limits from the phase 1 through 3 of the Tokyo axion helioscope experiments $[9,10,11]$ and some other bounds are also plotted in the same figure.

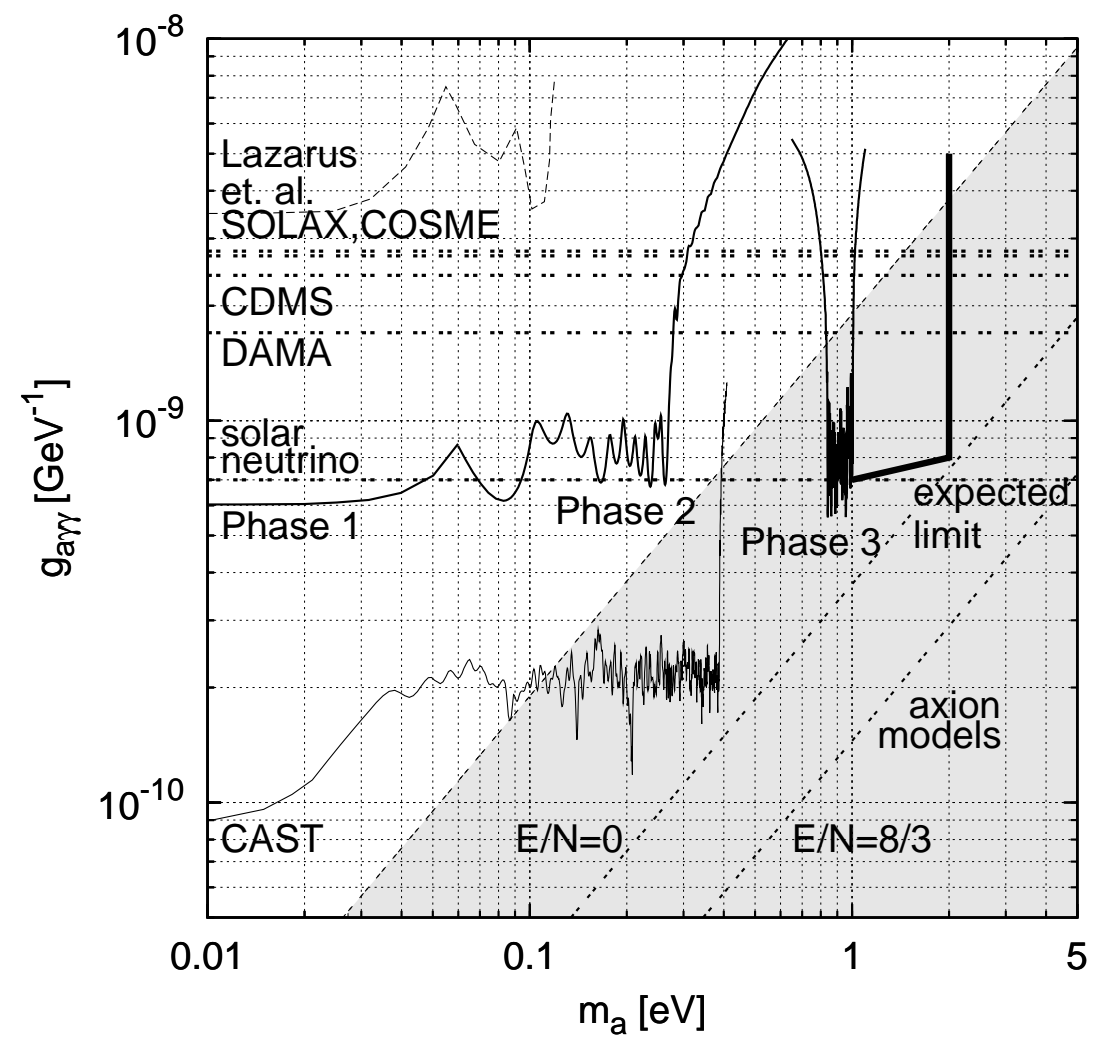

FIGURE 2. Exclusion plot on $g_{a \gamma \gamma}$ vs $m_{a}$.

The SOLAX [17], COSME [18] and DAMA [19] are solar axion experiments which exploit the coherent conversion on the crystalline planes [20] in a germanium and a NaI detector. Also shown is the solar limit inferred from the solar neutrino flux consideration[21]. The experiment by Lazarus et al. [6] and CAST [12, 13] are the same kind of helioscope experiments as mentioned above. The latter utilizes large decommissioned magnets of the LHC at CERN. Its limit is better than our previous limits by a factor of four to seven due to its higher magnetic field and longer field region. 
However, the upper limits around $1 \mathrm{eV}$ by Tokyo axion helioscope phase 3 measurement is a unique result in this mass region.

\section{PROSPECTS}

We are now preparing the search for solar axion with mass over $1 \mathrm{eV}$ introducing higher density helium gas than that of phase 3 . Figure 2 shows the expected upper limit of next measurement.

We are also constructing an additional detection unit on the Tokyo axion helioscope to search for hidden sector photons. The hidden sector photon is another kind of weakly interacting particles. Existence of the hidden sector photon is predicted by several extensions of the standard model. If light hidden sector photons exist, they could be produced through kinetic mixing with solar photons $[22,23]$. Therefore it is natural to consider the Sun as a source of low energy hidden photons. A detection schematics of hidden photons from the sun is shown in Fig. 3. Unlike the case of the axion, no magnetic field is required to transform hidden sector photons into photons.

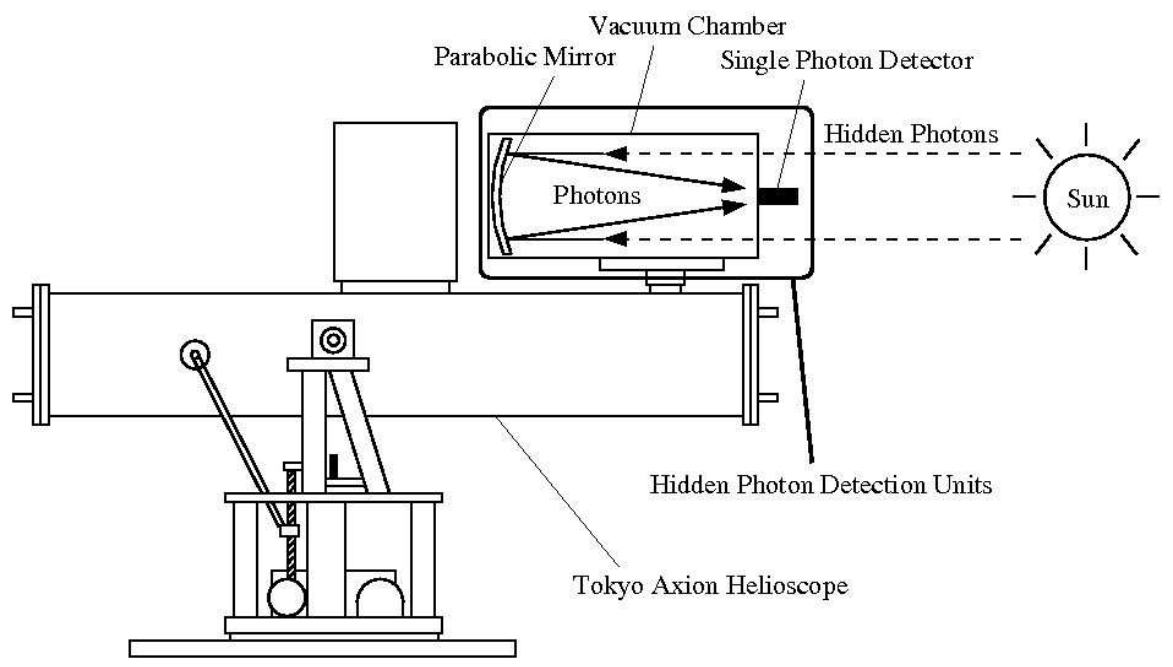

FIGURE 3. The schematic view of the apparatus to search for hidden photons from the sun.

\section{ACKNOWLEDGMENTS}

The author thanks the former director general of KEK, Professor H. Sugawara, for his support in the beginning of the helioscope experiment and all the collaboration members of Tokyo axion helioscope. This research was partially supported by the Japanese Ministry of Education, Science, Sports and Culture, Grant-in-Aid for COE Research and Grant-in-Aid for Scientific Research (B), and also by the Matsuo Foundation. 


\section{REFERENCES}

1. R.D. Peccei, H.R. Quinn, Phys. Rev. Lett. 38 (1977) 1440 and R.D. Peccei, H.R. Quinn, Phys. Rev. D 16 (1977) 1791.

2. S. Weinberg, Phys. Rev. Lett. 40 (1978) 223.

3. F. Wilczek, Phys. Rev. Lett. 40 (1978) 279.

4. J.E. Kim, Phys. Rep. 150 (1987) 1.

5. P. Sikivie, Phys. Rev. Lett. 51 (1983) 1415 and Phys. Rev. Lett. 52 (1984) 695.

6. D. M. Lazarus et al., Phys. Rev. Lett. 69 (1992) 2333.

7. K. Van Bibber et al., Phys. Rev. D 39 (1989) 2089.

8. G. Raffelt and L. Stodolsky, Phs. Rev. D 37 (1988) 1237.

9. S. Moriyama et al., Phys. Lett. B 434 (1998) 147.

10. Y. Inoue et al., Phys. Lett. B 536 (2002) 18.

11. Y. Inoue et al., Phys. Lett. B 668 (2008) 93.

12. K. Zioutas et al., Phys. Rev. Lett. 94 (2005) 121301, C. Eleftheriadis, arXiv:0706.0637v1, and S. Andriamonje et al. (CAST Collaboration), J. Cosmol. Astropart. Phys. 04 (2007) 010.

13. E. Arik, et al. (CAST Collaboration), J. Cosmol. Astropart. Phys. 02 (2009) 008.

14. Y. Sato et al., Development of a Cryogen-free Superconducting Dipole Magnet, in: Proc. of the 15th International Conference on Magnet Technology (MT-15) (Beijing, October 1997), eds.: L. Liangzhen, S. Guoliao, Y. Luguang (Science Press, Beijing, 1998) pp. 262-265; KEK-Preprint-97202 (November, 1997).

15. T. Namba et al., Nucl. Instr. Meth. A 489 (2002) 224.

16. Y. Akimoto et al., Nucl. Instr. Meth. A 557 (2006) 684.

17. A. O. Gattone et al., Nucl. Phys. B(Proc. Suppl.) 70 (1999) 59.

18. A. Morales et al., Astropart. Phys. 16 (2002) 325.

19. R. Bernabei et al., Phys. Lett. B 515 (2001) 6.

20. E. A. Paschos, K. Zioutas, Phys. Lett. B323 (1994) 367.

21. Paolo Gondolo and Georg Raffelt, Phys. Rev., D79 (2009) 107301.

22. Javier Redondo, J. Cosmol. Astropart. Phys. 07 (2008) 008.

23. Sergei N. Gninenko and Javier Redondo, Phys. Lett., B 664 (2008) 180. 